\section{Consolidated checklist of birds in the Pallikaranai Wetlands, Chennai, India}

\author{
P.P. Nikhil Raj ${ }^{1}$, J. Ranjini ${ }^{2}$, R. Dhanya ${ }^{3}$, \\ J. Subramanian ${ }^{4}$, P.A. Azeez ${ }^{5}$ \& S. Bhupathy ${ }^{6}$
}

1,2,3,5,6 Environment Impact Assessment Division, Sálim Ali Centre for Ornithology and Natural History (SACON), Anaikatty (PO), Coimbatore, Tamil Nadu 641108, India

${ }^{4}$ Pondicherry Central University, Kalapet, Puducherry 605014, India

Email: ${ }^{1}$ ppnraj@gmail.com, ${ }^{2}$ jranjini@gmail.com, ${ }^{3}$ dhanyajr@ gmail.com, ${ }^{5}$ azeezpa@gmail.com, ${ }^{6}$ bhupathy.s@gmail.com

Pallikaranai wetlands falls between $12^{0} 59^{\prime} \mathrm{N} \& 80^{\circ} 14^{\prime} \mathrm{E}$ (Fig. 1), located about $20 \mathrm{~km}$ south of the Chennai metropolitan area and falls in the Kancheepuram District of Tamil Nadu. The wetland runs along the old Mahabalipuram road parallel to the Buckingham Canal throughout its length in the south west of Chennai. The wetland is an extensive low lying area, covered by a mosaic of aquatic grass species, scrub, marsh, and water logged depressions. It has a catchment of $235 \mathrm{~km}^{2}$ that includes the urban sprawls of Velachery, Pallikaranai and Navalur. The wetland drains through Okkium Maduvu in Thoraipakkam to the Buckingham Canal, which discharges into the Kovalam Estuary (Care Earth 2002). The area receives $1300 \mathrm{~mm}$ of annual rainfall (www.wikipedia. com). The most rainfall occurs during the northeast monsoon (September - November) and partially from the

Date of publication (online): 26 July 2010

Date of publication (print): 26 July 2010

ISSN 0974-7907 (online) | 0974-7893 (print)

Editor: Rajah Jayapa

Manuscript details:

Ms \# 02220

Received 22 May 2009

Final revised received 16 June 2010

Finally accepted 29 June 2010

Citation: Raj, P.P.N., J. Ranjini, R. Dhanya, J. Subramanian, P.A. Azeez \& S. Bhupathy (2010). Consolidated checklist of birds in the Pallikaranai Wetlands, Chennai, India. Journal of Threatened Taxa 2(8): 1114-1118.

Copyright: @ P.P. Nikhil Raj, J. Ranjini, R. Dhanya, J. Subramanian, P.A Azeez \& S. Bhupathy 2010. Creative Commons Attribution 3.0 Unported License. JoTT allows unrestricted use of this article in any medium for non-profit purposes, reproduction and distribution by providing adequate credit to the authors and the source of publication.

Acknowledgements: We thank individually all the contributors of information for this checklist, SACON for the institutional support. We are grateful to the valuable help provided by our field assistants Mr. Raja and Mr. Marimuthu during the field work. We are also thankful to the reviewers for their valuable and reasonable comments which made further improvement of the manuscript. The paper is a tribute to Dr. Ravisankaran (Late). The authors are thankful to Dr. Sundaramurthy, CPR Foundation, Chennai.

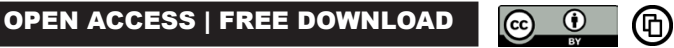

southwest monsoon (June - August). Temperatures vary seasonally; in summer the maximum ranging from $35^{\circ}$ to $42^{\circ} \mathrm{C}$ and in winter from $25^{\circ}$ to $34^{\circ} \mathrm{C}$ (Patnaik \& Srihari 2004).

A study conducted by Care Earth in 2002, supported by the Tamil Nadu Pollution Control Board (TNPCB) found that the area has reduced to about 600ha from an earlier extend of 4000-5000 ha (Care Earth 2002; Joint Committee Report 2003; The Hindu 2006). Further it has reduced to 420 ha (Care Earth 2005). A fall of about $30 \%$ in the wetland area within a span of three years is indeed alarming. The Chennai metropolis waste dump yard occupies a part of these wetlands, and it has effectively choked over 250 acres of prime marsh lands. In spite of the legalized and illicit encroachments, fast developing real estate ventures and various other developments including information technology parks on the banks of the marsh lands has aggravated the wetland depletion (Azeez et al. 2007a).

Realizing the environmental and ecological significance of the area in an expanding metropolis, the government of Tamil Nadu (Gazette notification GO. Ms. No. 52, dated 9 April 2007), declared a part of the Pallikaranai marsh (317.00ha) as a reserve forest (under section 4 of the Tamil Nadu Forest Act, 1882) in order to stall further conversion and protect the urban wetland (Azeez et al. 2007b). Subsequently, systematic and serious action plans are underway to conserve and rejuvenate this wetland ecosystem in a sustained manner. As a part of these efforts, we made a checklist of bird species of the Pallikaranai marshlands.

Methods: The study was conducted during February to August 2007 with two intensive surveys carried out for seven and 10 days respectively. The surveys covered the marsh land extending to the adjacent areas that included residential areas and walled campuses. Field survey was done during 0600 to $1000 \mathrm{hr}$ and 1700 to $1900 \mathrm{hr}$ adopting the line-transect method (Burnham et al. 1980; Bibby et al. 1992; Buckland et al. 1993). The opportunistic counts during other times of the day are also included in the checklist. Birds were recorded by direct sighting and calls. We followed the nomenclature and taxonomic sequence of Grimmett et al. (2000). The status of birds was categorized as Breeding Resident (BR), Winter Visitor (WV) and Vagrant (V) based on Grimmett et al. (2000). We also referred to the published and unpublished surveys conducted by different organizations and individuals in the past to complement our checklist (mainly Care Earth 2005, and unpublished work of the fourth author conducted during the year 2000).

Result and Discussion: In total, 101 species of resident and migratory birds were recorded from the Pallikaranai marshlands (Appendix 1). Of the total birds seen, 76 species were breeding residents and 25 winter 


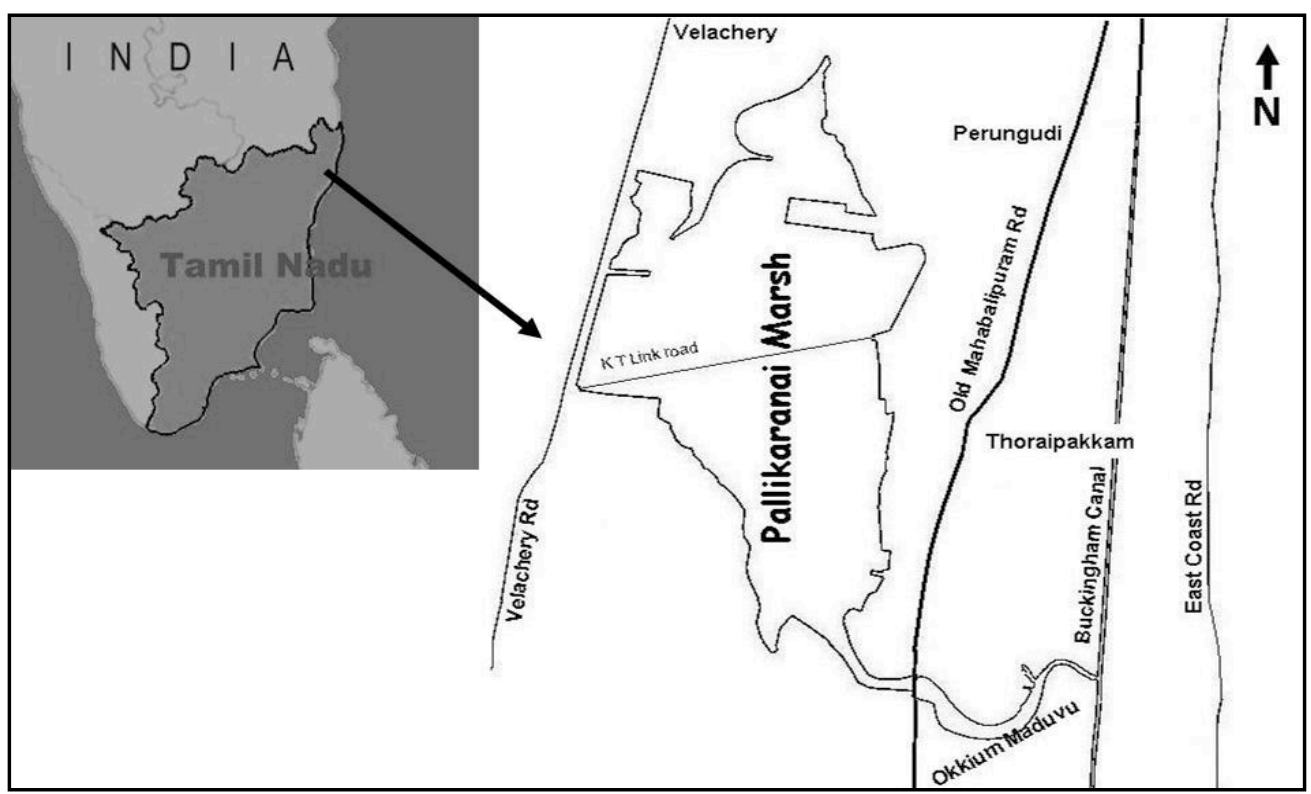

Figure 1. Map of Pallikaranai marsh

visitors. 40 species were fully aquatic, while 10 were partly dependent on wetlands. Among these species, the most numerous in our records included Little Grebe (ca.700) and Black-winged Stilt (ca.150). In a study conducted during the year 2000, 836 Black-winged Stilts and 834 Cattle Egrets were recorded (J. Subramanian pers. obs.). The Pallikaranai marshlands also include two Near Threatened bird species such as Spot-billed Pelican Pelecanus philippensis and Black-headed Ibis Threskiornis melanocephalus. During our surveys we found one Spot-billed Pelican in the wetlands; however, The Hindu released a report on 06 February 2007 with sightings of nearly 200 individuals in the Pallikaranai marshes. We could observe nearly 70 Black-headed Ibis, another Near Threatened species during the survey. The diversity of avifauna that occur in large numbers here calls for conservation of the Pallikaranai wetlands and prevention from further degradation so that it sustains as wetland for posterity offering the metropolis many ecological services. Conservation of such urban wetlands is essential to sustain migratory bird populations, as it is probably an abode during their migratory route, serving a vital role in the conservation of these species. Unfortunately, there is no laws till date to protect urban wetlands in particular, and we highlight here the urgent need for a policy to conserve urban wetlands and related ecosystems.

\section{REFERENCE}

Azeez P.A., S. Bhupathy, J. Ranjini, R. Dhanya \& P.P.N. Raj (2007). Management Plan for the Eco-restoration of Pallikaranai Reserve Forest. Salim Ali Centre for Ornithology and Natural History, Coimbatore, 62pp.
Bibby, C.J., N.D. Burgess \& D.A. Hill (1993). Bird Census Techniques. Academic Press Limited, London, 257pp.

BirdLife International (2001). Threatened Birds of Asia: The Bird Life International Red Data Book. Cambridge, UK, 864pp. http://www.birdlife.org/action/science/species/ global_species_programme/whats_new.html, downloaded on 16.11.2009.

Care Earth (2002). Conservation of Urban Wetlands - Pallikaranai Marsh. Tamil Nadu Pollution Control Board, 31pp.

Grimmet, R., C. Inskipp \& T. Inskipp (2000). Pocket Guide to the Birds of the Indian Subcontinent. Oxford University Press, New York, 384pp.

Joint Committee Report (2003). Threats to Pallikaranai wetland and need for its immediate remedial measures. Joint Committee, Ministry of Environment and Forest, Government of India, New Delhi.

Patnaik, D.C. \& P. Srihari (2004). Wetlands - A development paradox: The dilemma of South Chennai, India. Available at SSRN: http://ssrn.com/abstract=591861 or doi:10.2139/ ssrn.591861

The Hindu (2006). The marshland gone waste (report by $\mathrm{J}$ Vencatesan). http:// www.thehindu.com/thehindu/ pp/2006/07/01/stories/006070100040100.htm. 
Appendix 1. Bird species recorded in and around Pallikaranai Marsh

\begin{tabular}{|c|c|c|c|c|}
\hline & Common name & Scientific name & Habitat & Status \\
\hline 1 & Little Grebe *\# & Tachybaptus ruficollis & A & $\mathrm{BR}$ \\
\hline 2 & Little Cormorant *\#^ & Phalacrocorax niger & A & $\mathrm{BR}$ \\
\hline 3 & Great Cormorant $\# \wedge$ & Phalacrocorax carbo & A & $\mathrm{BR}$ \\
\hline 4 & Spot-billed Duck \# & Anas poecilorhyncha & A & $\mathrm{BR}$ \\
\hline 5 & Grey Heron *\#^ & Ardea cinerea & $A$ & WV \\
\hline 6 & Purple Heron *\#^ & Ardea purpurea & A & $\mathrm{BR}$ \\
\hline 7 & Cattle Egret ${ }^{* \star \Lambda}$ & Bubulcus ibis & $\mathrm{D}$ & $\mathrm{BR}$ \\
\hline 8 & Great Egret $\# \wedge$ & Casmerodius albus & A & $\mathrm{BR}$ \\
\hline 9 & Intermediate Egret *\#^ & Mesophoyx intermedia & A & $\mathrm{BR}$ \\
\hline 10 & Little Egret ${ }^{* \# \Lambda}$ & Egretta garzetta & A & $\mathrm{BR}$ \\
\hline 11 & Indian Pond Heron *\#^ & Ardeola grayii & A & BR \\
\hline 12 & Black-crowned Night Heron *\# & Nycticorax nycticorax & A & $\mathrm{BR}$ \\
\hline 13 & Black Bittern \# & Dupetor flavicollis & A & $\mathrm{BR}$ \\
\hline 14 & Cinnamon Bittern \# & Ixobrychus cinnamomeus & A & $\mathrm{BR}$ \\
\hline 15 & Yellow Bittern $\#$ & Ixobrychus sinensis & A & WV \\
\hline 16 & Spot-billed Pelican * & Pelecanus philippensis & A & $\mathrm{BR}$ \\
\hline 17 & Asian Openbill \#^ & Anastomus oscitans & A & $\mathrm{BR}$ \\
\hline 18 & Woolly-necked Stork \# & Ciconia episcopus & A & $\mathrm{BR}$ \\
\hline 19 & Glossy Ibis *\# & Plegadis falcinellus & A & WV \\
\hline 20 & Black-headed Ibis *\# & Threskiornis melanocephalus & A & $\mathrm{BR}$ \\
\hline 21 & Black Kite $\# \wedge$ & Milvus migrans & $\mathrm{O}$ & $\mathrm{BR}$ \\
\hline 22 & Black-shouldered Kite ${ }^{* \# \wedge}$ & Elanus caeruleus & $\mathrm{O}$ & WV \\
\hline 23 & Brahminy Kite $\# \wedge$ & Haliastur indus & $\mathrm{O}$ & $\mathrm{BR}$ \\
\hline 24 & Pallid Harrier \# & Circus macrourus & $\mathrm{O}$ & WV \\
\hline 25 & Pied Harrier \# & Circus melanoleucos & $\mathrm{O}$ & WV \\
\hline 26 & Eurasian Marsh Harrier *\# & Circus aeruginosus & $\mathrm{D}$ & WV \\
\hline 27 & Shikra *\#^ & Accipiter badius & $\mathrm{O}$ & $\mathrm{BR}$ \\
\hline 28 & Common Kestrel \# & Falco tinnunculus & O & WV \\
\hline 29 & Common Moorhen *\#^ & Gallinula chloropus & A & BR \\
\hline 30 & Purple Swamphen ${ }^{\# \wedge}$ & Porphyrio porphyrio & A & $\mathrm{BR}$ \\
\hline 31 & Coot *\#^ & Fulica atra & A & $\mathrm{BR}$ \\
\hline 32 & Eurasian Thick-knee ${ }^{\# \wedge}$ & Burhinus oedicnemus & A & $\mathrm{BR}$ \\
\hline 33 & Great Thick-knee \# & Burhinus recurvirostris & A & $\mathrm{BR}$ \\
\hline 34 & Red-necked Phalarope ${ }^{\#}$ & Phalaropus lobatus & A & $\mathrm{BR}$ \\
\hline 35 & Pheasant-tailed Jacana *\#^ & Hydrophasianus chirurgus & A & BR \\
\hline 36 & Red-wattled Lapwing *\#^ & Vanellus indicus & D & $\mathrm{BR}$ \\
\hline 37 & Yellow-wattled Lapwing *\#^ & Vanellus malabaricus & $\mathrm{D}$ & $\mathrm{BR}$ \\
\hline 38 & Black-winged Stilt *\# & Himantopus himantopus & A & $\mathrm{BR}$ \\
\hline 39 & Wood Sandpiper \# & Tringa glareola & A & WV \\
\hline 40 & Marsh Sandpiper *\# & Tringa stagnatilis & A & WV \\
\hline 41 & Oriental Pratincole ${ }^{\#}$ & Glareola maldivarum & A & WV \\
\hline 42 & Black-tailed Godwit \# & Limosa limosa & A & WV \\
\hline 43 & White-breasted Waterhen ${ }^{* \# \wedge}$ & Amaurornis phoenicurus & A & $\mathrm{BR}$ \\
\hline 44 & Watercock \#^ & Gallicrex cinerea & A & WV \\
\hline
\end{tabular}




\begin{tabular}{|c|c|c|c|c|}
\hline & Common name & Scientific name & Habitat & Status \\
\hline 45 & Greater Painted-snipe ${ }^{\# \wedge}$ & Rostratula benghalensis & A & $\mathrm{BR}$ \\
\hline 46 & Little Ringed Plover *\#^ & Charadrius dubius & A & $\mathrm{BR}$ \\
\hline 47 & Grey Plover *\# & Pluvialis squatarola & $A$ & WV \\
\hline 48 & River Tern *\#^ & Sterna aurantia & A & $\mathrm{BR}$ \\
\hline 49 & Whiskered Tern \# & Chlidonias hybridus & $A$ & WV \\
\hline 50 & White-winged Tern \# & Chlidonias leucopterus & A & WV \\
\hline 51 & Gull-billed Tern \# & Gelochelidon nilotica & $A$ & WV \\
\hline 52 & Rock Pigeon *\#^ & Columba livia & $\mathrm{O}$ & $\mathrm{BR}$ \\
\hline 53 & Spotted Dove *\# & Streptopelia chinensis & $\mathrm{O}$ & $\mathrm{BR}$ \\
\hline 54 & Rose-ringed Parakeet \# & Psittacula krameri & $\mathrm{O}$ & $\mathrm{BR}$ \\
\hline 55 & Asian Koel *\# & Eudynamys scolopacea & $\mathrm{O}$ & $\mathrm{BR}$ \\
\hline 56 & Common Hawk Cuckoo \# & Hierococcyx varius & 0 & $\mathrm{BR}$ \\
\hline 57 & Pied Cuckoo \#^ & Clamator jacobinus & $\mathrm{O}$ & $\mathrm{BR}$ \\
\hline 58 & Spotted Owlet \# & Athene brama & $\mathrm{O}$ & $\mathrm{BR}$ \\
\hline 59 & Barn Owl \# & Tyto alba & $\mathrm{O}$ & $\mathrm{BR}$ \\
\hline 60 & Common Kingfisher *\#^ & Alcedo atthis & $\mathrm{O}$ & $\mathrm{BR}$ \\
\hline 61 & White-throated Kingfisher *\#^ & Halcyon smyrnensis & $\mathrm{D}$ & $\mathrm{BR}$ \\
\hline 62 & Pied Kingfisher *\#^ & Ceryle rudis & $\mathrm{D}$ & $\mathrm{BR}$ \\
\hline 63 & Indian Roller ${ }^{\# \wedge}$ & Coracias benghalensis & $\mathrm{O}$ & $\mathrm{BR}$ \\
\hline 64 & Blue-tailed Bee-eater \#^ & Merops philippinus & 0 & WV \\
\hline 65 & Green Bee-eater *\#^ & Merops orientalis & $\mathrm{O}$ & $\mathrm{BR}$ \\
\hline 66 & Hoopoe $\#$ & Upupa epops & $\mathrm{O}$ & $\mathrm{BR}$ \\
\hline 67 & House Swift \# & Apus affinis & $\mathrm{O}$ & $\mathrm{BR}$ \\
\hline 68 & Asian Palm Swift \#^ & Cypsiurus balasiensis & $\mathrm{O}$ & $\mathrm{BR}$ \\
\hline 69 & Black-rumped Flameback \# & Dinopium benghalense & $\mathrm{O}$ & $\mathrm{BR}$ \\
\hline 70 & Barn Swallow *\# & Hirundo rustica & 0 & WV \\
\hline 71 & Wire-tailed Swallow \#^ & Hirundo smithii & $\mathrm{O}$ & WV \\
\hline 72 & Ashy-crowned Sparrow Lark \# & Eremopterix grisea & $\mathrm{O}$ & $\mathrm{BR}$ \\
\hline 73 & Oriental Skylark \# & Alauda gulgula & 0 & $\mathrm{BR}$ \\
\hline 74 & Common Myna *\#^ & Acridotheres tristis & $\mathrm{O}$ & $\mathrm{BR}$ \\
\hline 75 & Brahminy Starling \# & Sturnus pagodarum & $\mathrm{O}$ & WV \\
\hline 76 & House Crow *\#^ & Corvus splendens & $\mathrm{O}$ & $\mathrm{BR}$ \\
\hline 77 & Large-billed Crow *\# & Corvus macrorhynchus & $\mathrm{O}$ & $\mathrm{BR}$ \\
\hline 78 & Rufous Treepie *\# & Dendrocitta vagabunda & $\mathrm{O}$ & $\mathrm{BR}$ \\
\hline 79 & Red-vented Bulbul *\#^ & Pycnonotus cafer & 0 & $\mathrm{BR}$ \\
\hline 80 & Red-whiskered Bulbul \# & Pycnonotus jocosus & $\mathrm{O}$ & $\mathrm{BR}$ \\
\hline 81 & Yellow-billed Babbler *\# & Turdoides affinis & $\mathrm{O}$ & $\mathrm{BR}$ \\
\hline 82 & Common Tailorbird *\# & Orthotomus sutorius & $\mathrm{O}$ & $\mathrm{BR}$ \\
\hline 83 & Ashy Prinia *\# & Prinia socialis & $\mathrm{O}$ & $\mathrm{BR}$ \\
\hline 84 & Plain Prinia *\# & Prinia inornata & $\mathrm{O}$ & $\mathrm{BR}$ \\
\hline 85 & Blyth's Reed Warbler *\# & Acrocephalus dumetorum & 0 & WV \\
\hline 86 & Zitting Cisticola *\#^ & Cisticola juncidis & $\mathrm{O}$ & $\mathrm{BR}$ \\
\hline 87 & Pied Bush Chat *\# & Saxicola caprata & $\mathrm{O}$ & $\mathrm{BR}$ \\
\hline 88 & Oriental Magpie Robin \# & Copsychus saularis & $\mathrm{O}$ & $\mathrm{BR}$ \\
\hline 89 & Orange-headed Thrush \# & Zoothera citrina & $\mathrm{O}$ & WV \\
\hline 90 & Yellow Wagtail *\#^ & Motacilla flava & $D$ & WV \\
\hline
\end{tabular}




\begin{tabular}{|c|c|c|c|c|}
\hline & Common name & Scientific name & Habitat & Status \\
\hline 91 & White-browed Wagtail \#^ & Motacilla maderaspatensis & 0 & BR \\
\hline 92 & Paddy-field Pipit ${ }^{*} \wedge$ & Anthus rufulus & D & BR \\
\hline 93 & Richard's Pipit \# & Anthus richardi & D & WV \\
\hline 94 & House Sparrow *\# & Passer domesticus & $\mathrm{O}$ & BR \\
\hline 95 & Streaked Weaver\# & Ploceus manyar & 0 & BR \\
\hline 96 & Black-headed Munia \# & Lonchura malacca & D & BR \\
\hline 97 & Black Drongo *\#^ & Dicrurus adsimilis & 0 & BR \\
\hline 98 & Ashy Woodswallow" & Artamus fuscus & 0 & BR \\
\hline 99 & Eurasean Golden Oriole \# & Oriolus oriolus & 0 & BR \\
\hline 100 & Loten's Sunbird \# & Nectarinia Iotenia & 0 & BR \\
\hline 101 & Grey Francolin ${ }^{\# \wedge}$ & Francolinus pondicerianus & 0 & BR \\
\hline
\end{tabular}

BR - Breeding Resident; V - Vagrant; WV - Winter Visitor; A - Aquatic; D - Water dependent; O - Others; * - Present study; \# - Care Earth 2002; ^ - J. Subramanian (Unpublished) 